\title{
Relativistic Vlasov-Uehling-Uhlenbeck model for heavy-ion collisions
}

\author{
Che Ming Ko and Qi Li \\ Cyclotron Institute and Center for Theoretical Physics, Texas A\&M University, \\ College Station, Texas 77843 \\ (Received 28 December 1987)
}

\begin{abstract}
The previously derived relativistic Vlasov equation from the Walecka model is generalized to include both a collision term and the self-interaction of the scalar meson. From studying the transverse momentum distribution in heavy-ion collisions, we conclude that the nuclear equation of state at high density is softer than that determined previously using the normal VlasovUehling-Uhlenbeck model and is consistent with that from the analysis with a momentumdependent potential. We have also found that in relativistic model the magnitude of the transverse momentum from heavy-ion collision is more sensitive to the value of the nucleon effective mass at saturation density than the value of the compressibility at this density.
\end{abstract}

To construct a theory for heavy-ion collisions at high energies when particles move with a velocity not negligible with respect to the velocity of light, it is important to take into account the relativistic effects. This includes the explicit mesonic-exchange nature of the interaction, the small component of the nucleon wave function, and the existence of the negative energy states. Also at very high density and/or temperature, the restoration of the chiral symmetry is expected to play an important role in highenergy heavy-ion collisions. As a first step towards the development of a relativistic transport theory for heavy-ion collisions which incorporates the above effects, we have recently derived a relativistic Vlasov equation from the Walecka model in the local-density and the semiclassical approximation. ${ }^{1}$ In the Walecka model, ${ }^{2}$ the interaction between the nucleons are mediated by a scalar meson $\phi$ with mass $m_{s}$ and a vector meson $V_{\mu}$ with mass $m_{v}$. Introducing the phase space distribution function $f(r, p)$ for the nucleons, then the following relativistic Vlasov equation has been obtained in Ref. 1:

$$
\partial_{t} f+\dot{\mathbf{r}} \cdot \nabla_{\mathrm{r}} f+\dot{\mathbf{p}} \cdot \nabla_{\mathrm{p}} f=0 \text {, }
$$

where

$$
\dot{\mathbf{r}}=\mathrm{p}^{*} / e^{*}
$$

and

$$
\dot{\mathbf{p}}=-\nabla_{r} U=\nabla_{r}\left(e^{*}+g_{v} V_{0}\right) .
$$

The coupling constants of the mesons to the nucleon are denoted by $g_{s}$ and $g_{v}$ for the scalar and the vector meson, respectively. In the above, $\mathrm{p}^{*}=\mathrm{p}-g_{v} \mathrm{~V}$ and $e^{*}=\left(\mathrm{p}^{* 2}\right.$ $\left.+M^{* 2}\right)^{1 / 2}$ with $M^{*}=M-g_{s} \phi$. The nucleon mass is denoted by $M$. In the mean-field approximation, the meson fields are treated as classical fields and are related to the nuclear densities via

$$
\sigma \approx\left(g_{s} / m_{s}^{2}\right) \rho_{s}, \quad V_{0} \approx\left(g_{v} / m_{v}^{2}\right) \rho_{B}, \quad \mathrm{~V} \approx\left(g_{v} / m_{v}^{2}\right) \rho_{v}
$$

if we neglect the dynamics of meson propagations. As pointed out in Ref. 1, this approximation is probably reasonable as both mesons have relatively large masses. It is certainly worthwhile in the future to investigate more closely its validity. In Eq. (2), the baryon density $\rho_{B}$, the scalar density $\rho_{s}$, and the current density $\rho_{\mathrm{v}}$ can be expressed in terms of the phase-space distribution function as follows:

$$
\begin{aligned}
& \rho_{B}(\mathrm{r})=\int d^{3} \mathrm{p} f(\mathrm{r}, \mathrm{p}), \\
& \rho_{s}(\mathbf{r})=\int d^{3} \mathrm{p}\left(M^{*} / e^{*}\right) f(\mathrm{r}, \mathrm{p}),
\end{aligned}
$$

and

$$
\rho_{\mathrm{v}}(\mathrm{r})=\int d^{3} \mathrm{p}\left(\mathrm{p}^{*} / e^{*}\right) f(\mathrm{r}, \mathrm{p}) .
$$

A covariant Vlasov equation has also been derived in Ref. 3 based on the Wigner distribution function. The equivalence of our equation to that in Ref. 3 is shown in Ref. 4. It is therefore immaterial whether Eq. (1) is solved in the laboratory frame or the center-of-mass frame of heavy-ion collisions. In Ref. 1, the relativistic Vlasov equation is solved using the method of the pseudoparticles. Applying it to heavy-ion collisions at intermediate energies, we have obtained results that are similar to those from the more complicated time-dependent Dirac equation. ${ }^{5}$ In particular, both lead to an appreciable transverse-momentum distribution during the collision.

In order to apply the relativistic model to high-energy heavy-ion collisions, it is necessary to include a collision term. Since a satisfactory derivation of the collision term is not yet available, we shall therefore use the collision integral as in the ordinary Vlasov-Uehlung-Uhlenbeck (VUU) model, ${ }^{6}$ i.e.,

$$
\begin{aligned}
I_{c}=\int & \frac{d^{3} \mathbf{p}_{2} d^{3} \mathbf{p}_{3} d \Omega}{(2 \pi)^{6}} v_{12} \sigma(\Omega)(2 \pi)^{3} \delta^{(3)}\left(\mathbf{p}_{1}+\mathbf{p}_{2}-\mathbf{p}_{3}-\mathbf{p}\right) \\
& \times\left\{f\left(\mathbf{p}_{1}, \mathbf{r}\right) f\left(\mathbf{p}_{2}, \mathbf{r}\right)\left[1-f\left(\mathbf{p}_{3}, \mathbf{r}\right)\right][1-f(\mathbf{p}, \mathbf{r})]-f(\mathbf{p}, \mathbf{r}) f\left(\mathbf{p}_{3}, \mathbf{r}\right)\left[1-f\left(\mathbf{p}_{1}, \mathbf{r}\right)\right]\left[1-f\left(\mathbf{p}_{2}, \mathbf{r}\right)\right]\right\}
\end{aligned}
$$


The essential ingredients in this collision integral are the effective nucleon-nucleon cross section $\sigma(\Omega)$ and the Pauli-blocking factors for the phase space as shown by $\{\cdots\}$ in the above equation. As to the effective nucleonnucleon cross section, we shall use that in the free space. The medium effect on the nucleon-nucleon cross section besides that of the Pauli principle is currently controversial. Using the relativistic Brueckner theory, ter Haar and Malfliet ${ }^{7}$ have obtained an effective cross section which is about $30 \%$ smaller than its value in the free space. On the other hand, Brown ${ }^{8}$ claims that it should be a factor of 2 larger if the polarization effect on the mesons is taken into account.

The original Walecka mean-field model gives a nuclear compressibility of $540 \mathrm{MeV}$ which is much larger than that determined from the energy of the giant monopole resonances in nuclei. It also leads to a nucleon effective mass of $0.56 \mathrm{M}$ at the saturation density which is smaller than the value determined by the optical model analysis of nucleon-nucleus scattering. ${ }^{9}$ In order to have a model that allows for various values of the nuclear compressibility and the effective nucleon mass, we shall include the self-interaction of the scalar meson, i.e.,

$$
U(\phi)=b \phi^{3} / 3+c \phi^{4} / 4 \text {. }
$$

The two parameters $b$ and $c$ make it possible to obtain different values of the compressibility and the effective nucleon mass as shown in Ref. 10. With the self-interaction of the scalar meson, the relation between the value of the scalar meson and the nuclear scalar density in Eq. (2) becomes

$$
m_{s}^{2} \phi+b \phi^{2}+c \phi^{3} \approx g_{s} \rho_{s}
$$

We shall apply the relativistic model to study the highenergy heavy-ion collisions. At such high energies, pion production plays an important role. To include such effects, we allow the nucleon to be excited to the delta resonance in the collision. When two nucleons undergo a collision, the probability for nucleon to change into a delta is determined by the ratio of the nucleon-nucleon inelastic cross section to the total cross section as in the cascade model. ${ }^{11}$ However, we neglect the decay of the delta resonance into the pion as we do not know yet how to include properly pions in the relativistic theory. From the cascade model, ${ }^{11}$ it has been shown that pions appear mostly in the later stage of the collision and will probably not affect appreciably the initial dynamics which is what we are mainly interested in. Once delta is created, its dynamic will follow a similar equation as the nucleon, i.e., Eqs. (1) to (4). To determine the mean field for the delta resonance in the relativistic model, we need to know its couplings to the scalar and the vector mesons which we take to be the same as those of the nucleon.

We have studied the reaction ${ }^{40} \mathrm{Ca}+{ }^{40} \mathrm{Ca}$ at an incident energy of $1.8 \mathrm{GeV} /$ nucleon $^{12}$ and at an impact parameter of $2 \mathrm{fm}$ corresponding to a central collision. We solve Eqs. (1) and (2) using the method of test particles. In the numerical simulations, each nucleon is replaced by 80 test particles and at least ten simulations are carried out for each case. The time interval used for evolving the system is taken to be $0.5 \mathrm{fm} / c$. We have also used a smaller time step of $0.2 \mathrm{fm} / c$ in a few cases and found no significant difference from the results obtained with a time step of 0.5 $\mathrm{fm} / c$. The nucleon transverse momentum distribution after the collision has been measured. An earlier study of this reaction with the normal VUU model has led to the conclusion that the nuclear equation of state is rather stiff with a compressibility of $380 \mathrm{MeV} .{ }^{13}$ This equation of state, expressed as the binding energy per nucleon as a function of the nuclear density, is shown in Fig. 1 by the long-dashed curve. We have calculated the transverse momentum distribution for this reaction with the same compressibility but with an effective nucleon mass of $0.83 M$ which is suggested by the latest determination by Johnson, Horen, and Mahaux from the optical model analysis of nucleon-nucleus scattering. ${ }^{9}$ To obtain these values of the compressibility and the nucleon effective mass, we use the following values for the parameters in the generalized Walecka model:

$$
\begin{aligned}
& C_{v}=\left(g_{v} / m_{v}\right) M=9.04, C_{s}=\left(g_{s} / m_{s}\right) M=11.78, \\
& B=b /\left(g_{s}^{3} M\right)=-2.59 \times 10^{-2}, C=c / g_{s}^{4}=0.169,
\end{aligned}
$$

if a binding energy of $15.96 \mathrm{MeV}$ at a saturation density $0.145 \mathrm{fm}^{-3}$ is required. The corresponding equation of state is given by the solid curve in Fig. 1 and is seen to be much softer than the previous one at high densities. The transverse momentum distribution as a function of the rapidity calculated from the relativistic model with the parameters given by Eq. (7) is shown in Fig. 2 by the solid curve. It agrees reasonably with the experimental data as shown by the open diamonds. The errors in our results are only about $5 \%$ except at small rapidities where they are about $15 \%$ due to the small number of test particles in this rapidity region. These errors can be further reduced if more simulations are carried out. When a momentumdependent potential is included in the normal VUU mod-

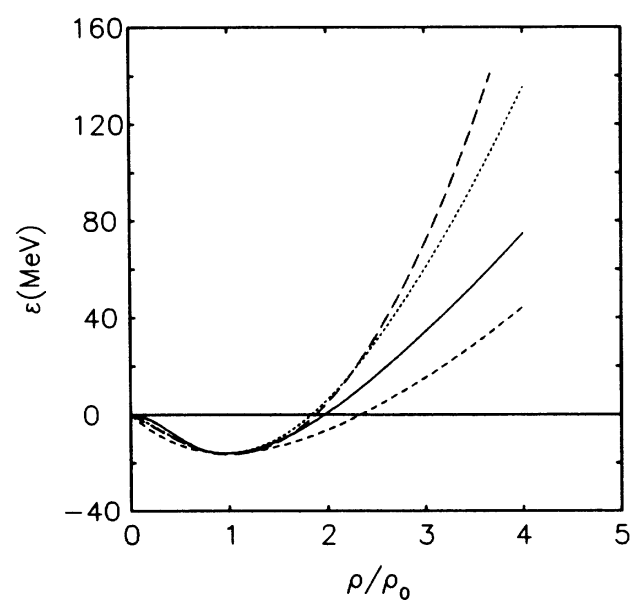

FIG. 1. The binding energy per nucleon $\varepsilon$ as a function of the ratio of the density to the saturation density $\rho / \rho_{0}$ for the three cases: solid curve $\left(M^{*}=0.83 M, K=380 \mathrm{MeV}\right)$, dashed curve $(0.83 M, 200 \mathrm{MeV})$, and dotted curve $(0.7 M, 380 \mathrm{MeV})$. The long-dashed curve corresponds to a stiff equation of state used in the normal VUU model (Ref. 13). 




FIG. 2. The transverse momentum projected onto the reaction plane and averaged over all perpendicular momentum $\boldsymbol{P}_{\boldsymbol{x}}$, as a function of the rapidity $y$ for the reaction ${ }^{40} \mathrm{Ca}+{ }^{40} \mathrm{Ca}$ at 1.8 $\mathrm{GeV} /$ nucleon. The open diamonds are the experimental data from Ref. 12. The solid curve and the dashed curve are from the theoretical calculations with the same effective mass $M^{*}=0.83 M$ but different values of the compressibility $K=380$ $\mathrm{MeV}$ and $K=200 \mathrm{MeV}$, respectively. The dotted curve corresponds to $M^{*}=0.7 M$ and $K=380 \mathrm{MeV}$.

el, a similar result can be obtained for the transverse momentum distribution with a compressibility of about $200 \mathrm{MeV} .{ }^{14,15}$ We have also carried out calculations with this value of the compressibility and an effective nucleon mass $0.83 M$. This equation of state is shown by the dashed curve in Fig. 1 and is obtained with the parameters, $C_{v}=9.04, \quad C_{s}=14.5, \quad B=1.73 \times 10^{-2}, \quad$ and $C=-1.20 \times 10^{-3}$. The resulting transverse momentum distribution is shown in Fig. 2 by the dashed curve and is similar to the previous one obtained with a compressibility of $380 \mathrm{MeV}$. We therefore conclude that in the relativistic model the transverse momentum distribution in highenergy heavy-ion collisions is not sensitive to the value of the compressibility.

To understand the above result, we note that the final transverse momentum distribution is essentially reached at the highest compression in the collision when the maximum density is about 2.7 times the normal nuclear matter density in the above example. The pressure responsible for the particles to move sideways is determined by the density dependence of the nuclear part of the single-particle energy as shown in Eq. (1c). For heavy-ion collisions at high energies, the density dependence of the nucleon kinetic energy through the nucleon effective mass is small in comparison with the density dependence of the potential energy due to the interaction with the vector meson. Since the vector meson coupling constant is fixed by the value of the nucleon effective mass, the pressure on the nucleons is similar for the two values of compressibility. The apparent stiffness of the nuclear equation of state at high densities for a larger value of compressibility has thus no direct effect on the transverse momentum distribution for high-energy heavy-ion collisions. At lower incident energies, the density dependence of the nucleon ki-

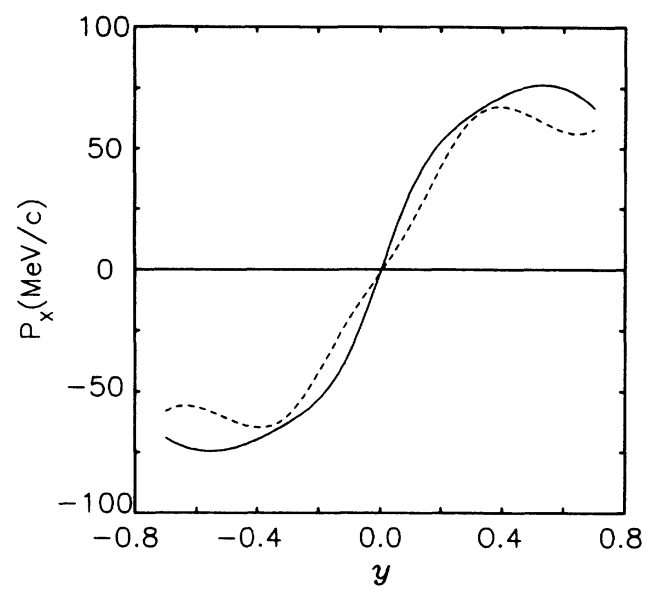

FIG. 3. Same as Fig. 2 for an incident energy of 800 $\mathrm{MeV} /$ nucleon. The solid curve and the dashed curve are from the theoretical calculations with the same effective mass $M^{*}=0.83 M$ but different values of compressibility $K=380$ $\mathrm{MeV}$ and $200 \mathrm{MeV}$, respectively.

netic energy will be appreciable and the transverse momentum distribution is therefore expected to be sensitive to the value of the compressibility. To demonstrate this effect, we have repeated the above calculations for an incident energy of $800 \mathrm{MeV} /$ nucleon and the results for the transverse momentum distribution are shown in Fig. 3 for the two equations of state. We indeed see a larger transverse momentum for the stiffer equation of state (solid curve) than the softer equation of state (dashed curve). The maximum density reached during the collision at this energy is lower than the higher energy case and has a value of about 2.6 times the normal nuclear matter density.

On the other hand, the transverse momentum distribution changes drastically if a different value of the nucleon effective mass is used as shown by the dotted curve in Fig. 2 which corresponds to an effective mass of $0.7 M$ and a compressibility of $380 \mathrm{MeV}$. The corresponding equation of state is given by the dotted curve in Fig. 1 and is obtained by using the parameters, $C_{v}=13.0, C_{s}=16.05$, $B=-1.69 \times 10^{-4}$, and $C=5.18 \times 10^{-3}$. The reason for this is because a smaller effective mass implies a larger value of the vector meson coupling constant which leads thus to a stronger sideways pressure on the particles. The maximum density reached in the collision is about 2.4 times the normal nuclear matter density and is smaller than the case with an effective nucleon meson of $0.83 M$.

In summary, we have applied the relativistic transport model to study the transverse momentum distribution in heavy-ion collisions. From comparing with the available experimental data, it is concluded that a relatively soft equation of state is required. This result is similar to that from previous studies with a momentum-dependent potential. In the relativistic model, the magnitude of the transverse momentum from heavy-ion collisions is more sensitive to the value of the nucleon effective mass at saturation density than the value of the compressibility at this density. 
We are grateful to Norman Glendenning for bringing to our attention the recent work of Johnson, Horen, and Mahaux on the nucleon effective mass. This work is supported in part by the National Science Foundation under Grant No. 8608149 and the Robert A. Welch Foundation under Grant No. A-1110.

${ }^{1}$ C. M. Ko, Q. Li, and R. Wang, Phys. Rev. Lett. 59, 1084 (1987)

2J. D. Walecka, Ann. Phys. 83, 491 (1974).

${ }^{3}$ H. Elze et al., Mod. Phys. Lett. 2, 451 (1987).

${ }^{4} \mathrm{Q}$. Li and C. M. Ko, Mod. Phys. Lett. (to be published).

${ }^{5}$ R. Y. Cusson et al., Phys. Rev. Lett. 55, 2786 (1985).

${ }^{6}$ G. F. Bertsch, H. Kruse, and S. Das Gupta, Phys. Rev. C 29, 673 (1984).

${ }^{7}$ B. ter Haar and R. Malfliet, Phys. Rep. 149, 207 (1987).

${ }^{8}$ G. E. Brown, in Proceedings of the Eighth High Energy Heavy Ion Study, Berkeley, California, 1987 (unpublished).
${ }^{9}$ C. H. Johnson, D. J. Horen, and C. Mahaux, Phys. Rev. C 36, 2252 (1987).

10J. Boguta and H. Stöcker, Phys. Lett. 120B, 289 (1983).

11 J. Cugnon, D. Kinet, and J. Vandermeulen, Nucl. Phys. A379, 553 (1982).

${ }^{12}$ H. Ströbele et al., Phys. Rev. C 27, 1349 (1983).

${ }^{13}$ J. J. Molitoris and H. Stöcker, Phys. Rev. C 32, 346 (1985).

${ }^{14}$ J. Aichelin et al., Phys. Rev. Lett. 58, 1926 (1987).

${ }^{15}$ C. Gale, G. Bertsch, and S. Das Gupta, Phys. Rev. C 35, 1666 (1987). 